\title{
Cenobamate-The New Kid on the Block, Teaching More About Epilepsy Than Just Offering a New Treatment for Seizures
}

\author{
Roy G Beran ${ }^{1-3}$
}

1. University of New South Wales, Sydney, New South Wales, Australia; 2. School of Medicine, Griffith University, Queensland, Australia;

3. Sechenov First Moscow State Medical University, Moscow, Russia

DOI: https://doi.org/10.17925/USN.2020.16.2.85

$\mathrm{T}$ e treatment of epilepsy is evolving, and with it, so should our approach to treatment. A recent article by Klein et al. (published in this issue of US Neurology) confronted my own attitude towards anti-epileptic medication, and ultimately changed the way I view this therapy and the nomenclature I use. Their empathetic approach to the topic of new anti-seizure medication compelled me to write something that is normally outside of my usual remit. I hope this first-person perspective reflects the impact this article had on me and encourages others to consider their own philosophy in this field.

\section{Keywords}

Epilepsy, anti-seizure medication, placebo effect, cenobamate

Disclosure: Roy G Beran has no financial or non-financial relationships or activities to declare in relation to this article.

Review Process: Double-blind peer review.

Compliance with Ethics: This article is an opinion piece and does not report on new clinical data, or any studies with human or animal subjects performed by the author.

Authorship: The named author meets the International Committee of Medical Journal Editors (ICMJE) criteria for authorship of this manuscript, takes responsibility for the integrity of the work as a whole, and has given final approval for the version to be published.

Access: This article is freely accessible at

tOUChNEUROLOGY.com (C) Touch Medical Media 2020

Received: July 6, 2020

Accepted: September 23, 2020

Published Online: December 12, 2020

Citation: US Neurology. 2020;16(2):85-6

Corresponding Author: Roy G Beran, Suite 5, Level 6, 12 Thomas Street, Chatswood, NSW 2067, Australia.

E: roy@royberan.com

Support: No funding was received in

the publication of this article.
Dogma dictates that scientific literature should be couched in the third person, past tense. The idea is to obviate the potential to introduce personal bias that may accompany first person, present tense, which is creeping into modern scientific writing. This use of the first person, I am reliably told, followed wide adoption of blogging, in which the authors assume the authority to share personal views without the prerequisite for literature support to underpin that which the blogger may espouse.

As a pedant of scientific writing, it has been a personal approach to retain third person, past tense; but to every rule, there is an exception. The article entitled, "Cenobamate for the Treatment of Focal Seizures", by Klein et al., which appears in this issue of US Neurology, evoked a feeling of empathy and dealt with issues that transcend a simple review of yet another new medication, namely cenobamate, in the management of people with epilepsy. ${ }^{1}$ It led me to offer the provision of an editorial that is more akin to a blog, and will allow the introduction of personal bias, including first-person syntax.

Over the years, I have chosen to avoid the acronym, AED, to denote "anti-epileptic drug", and preferred AEM to reflect "anti-epileptic medication". This removed the negative connotation attached to drugs and the current overt battle against drugs. Accepting that the concept of drugs produces negative implications, it seemed inappropriate to expand the stigma that is attached to epilepsy to include the potential concept of drug dependence; acknowledging that people with epilepsy are dependent on medication(s) to control their seizures. In their abstract, Klein et al. have taken this one step further. They have acknowledged that the medications used in the treatment of epilepsy are not anti-epileptic (a concept incorporated into the acronym, AED, and further incorporated in the name of the professional body - the International League Against Epilepsy), but are used to treat epilepsy and are designed to stop seizures. Klein et al. introduced a more appropriate and acceptable term, "anti-seizure medication" (ASM). This fosters a further move away from the concept of drugs and the associated negative implications. I fully endorse Klein et al's use of the acronym, ASM, in preference to my own use of AEM, as it better reflects that which the medications are designed to achieve. I sincerely hope that ASM becomes the chosen, and accepted, acronym to denote these medications. It is appropriate that the introduction of this acronym is attached to this review of the latest arrival in the treatment against seizures.

A second feature that permeates the paper by Klein et al., is the acknowledgment of a new classification of seizures. ${ }^{2,3}$ It has been shown that many clinicians are unaware of these classifications, be it the older classification or the newer terminology. ${ }^{4}$ In their paper, Klein et al. have transcended the gulf between old and new classifications and have adopted both terminologies within the text, such as focal aware (simple partial onset), focal impaired awareness (complex partial onset), and focal to bilateral tonic-clonic (secondarily generalized tonic-clonic) seizures. This allows those who are less 
familiar with the latest terminology to also update their knowledge of epilepsy while concurrently learning about cenobamate.

Having identified the politically correct terminology adopted by Klein et al., there is a third point to be drawn from this paper. This is above and beyond the principal take-home message, which is that cenobamate offers a novel, exciting, and effective additional tool with which to manage seizures and to improve the quality of life for people with epilepsy. The paper by Klein et al. raises the major distinguishing feature of cenobamate, compared with other ASMs introduced over the last 30 years, namely its exceptional efficacy, including $20 \%$ seizure freedom in patients with drug-resistant focal epilepsy. This was further emphasized, when, reporting the efficacy of cenobamate, it was shown that there was a $55.6 \%$ median reduction in seizures versus a $21.5 \%$ reduction for patients on placebo. Breaking this down into seizure types, it translated to $76.3 \%$ versus $27.8 \%$ for focal aware (simple partial seizures), $55.6 \%$ versus $21.1 \%$ for focal impaired awareness (complex partial) seizures, and $77.0 \%$ versus $33.04 \%$ for focal to bilateral tonic-clonic (secondarily generalized tonic-clonic) seizures. This confirms the statistically significant efficacy of cenobamate as an exciting addition to the available ASMs, especially over placebo, and demonstrates the ideal dosage to be between $200 \mathrm{mg}$ and $400 \mathrm{mg}$ daily; it also showed the impact of the placebo effect in treating epilepsy.

With cenobamate, being the "new kid on the block", it is to be assumed that the patients who were involved in the clinical trials were skewed toward those who experienced more difficult-to-control epilepsy. Upon this backdrop, up to one in three patients with focal to bilateral tonic-clonic seizures had an effective response when treated with placebo, almost $30 \%$ with focal aware seizures responded to placebo, and one in four focal awareness impaired seizures also responded to placebo. These numbers are very impressive, when considering the potentially refractory nature of the target population in these trials. Sodium valproate, a widely used ASM, was considered a placebo in clinical trials before being tested in its own right. 5,6 With this realization, it may behove the investigators to examine what was used as placebo in the cenobamate trials, and to consider if this may also provide the basis for another potential ASM.

It has been argued that this observation, regarding the placebo effect, may be an overstatement of reality within trials and may be an artificial aberration that is ill founded in the 21st Century. Goldenholz et al. have debated this point, arguing that it may be a reflection of the natural history of epilepsy; ${ }^{7}$ however, the figures for placebo effect remain impressive, and without an open and questioning mind we may miss the next valuable addition to our existing ASMs.

\section{Conclusion}

Klein et al. have achieved more than simply providing a report on the role and impact of cenobamate in the treatment of epilepsy. They introduced the more acceptable acronym, ASM, to denote anti-seizure medication, thereby removing any reference to drugs and highlighting that the medications deal with seizures, rather than epilepsy. They offered a workable transition to allow those less familiar with the terminology to move between the old and new classifications. Finally, they highlighted the potential for a definitive placebo effect in the treatment of epilepsy, which may possibly translate to further discovery and evolution of the pharmacology of ASMs, assuming anyone is prepared to pursue it when it may be an aberration, and depending upon the chemical formula and nature of the placebo used in these trials. $\square$

1. Klein P, Ferrari L, Rosenfeld WE. Cenobamate for the treatment of focal seizures. US Neurology. 2020;16:87-97

2. Fisher RS, Cross HJ, French JA, et al. Operational classification of seizure type by the International League Against Epilepsy: Position paper of the ILAE Commission for Classification and Terminology. Epilepsia. 2017:58:522-30.

3. Falco-Walter JJ, Scheffer IE, Fisher RS. The new definition and classification of seizures and epilepsy. Epilepsy Res. 2018;139:73-9.

4. Sharobeam A, Mamun A, Kwok A, et al. Management and attitudes towards patients with epilepsy in general practice: how far have we come in three decades? Epilepsy Behav. 2019;97:92-5.

5. Löscher W. The discovery of valproate. In: Löscher W (ed). Valproate. Milestones in Drug Therapy. Birkhäuser, Basel. 1999. Available at: https://doi.org/10.1007/978-3-0348-8759-5_1 (accessed December 21, 2020).

6. Tomson T, Battino D, Perucca E. The remarkable story of valproic acid. Lancet Neurol. 2016;15:141.

7. Goldenholz DM, Moss R, Scott J, et al. Confusing placebo effect with natural history in epilepsy: a big data approach. Ann Neurol. 2015;78:329-36. 